Open Access

\title{
Efficacy of supraspinatus tendon repair using mesenchymal stem cells along with a collagen I scaffold
}

Pilar Tornero-Esteban ${ }^{1}$, José Antonio Hoyas ${ }^{1}$, Esther Villafuertes ${ }^{1}$, Cruz Rodríguez-Bobada ${ }^{2}$, Yamila López-Gordillo ${ }^{3}$, Francisco J. Rojo ${ }^{4}$, Gustavo V. Guinea ${ }^{4,5}$, Anna Paleczny ${ }^{4,5}$, Yaiza Lópiz-Morales ${ }^{6}$, Luis Rodriguez-Rodriguez ${ }^{1}$ Fernando Marco ${ }^{6}$ and Benjamín Fernández-Gutiérrez ${ }^{1 *}$

\begin{abstract}
Objectives: Our main objective was to biologically improve rotator cuff healing in an elderly rat model using mesenchymal stem cells (MSCs) in combination with a collagen membrane and compared against other current techniques.

Methods: A chronic rotator cuff tear injury model was developed by unilaterally detaching the supraspinatus (SP) tendons of Sprague-Dawley rats. At 1 month postinjury, the tears were repaired using one of the following techniques: (a) classical surgery using sutures $(n=12)$, (b) type I collagen membranes $(n=15)$, and (c) type I collagen membranes $+1 \times 106$ allogeneic MSCs $(n=14)$. Lesion restoration was evaluated at 1,2 , and 3 months postinjury based on biomechanical criteria. Continuous variables were described using mean and standard deviation (SD). To analyse the effect of the different surgical treatments in the repaired tendons' biomechanical capabilities (maximum load, stiffness, and deformity), a two-way ANOVA model was used, introducing an interaction between such factor and time (1,2, and 3 months postinjury).

Results: With regard to maximum load, we observed an almost significant interaction between treatment and time ( $F=2.62, d f=4, p=0.053)$. When we analysed how this biomechanical capability changed with time for each treatment, we observed that repair with OrthADAPT and MSCs was associated with a significant increase in maximum load $(p=0.04)$ between months 1 and 3 . On the other hand, when we compared the different treatments among themselves at different time points, we observed that the repair with OrthADAPT and MSCs has associated with a significant higher maximum load, when compared with the use of suture, but only at 3 months $(p=0.014)$. With regard to stiffness and deformity, no significant interaction was observed $(F=1.68$, $d f=4, p=0.18 ; F=0.40, d f=4, p=0.81$; respectively).

Conclusions: The implantation of MSCs along with a collagen I scaffold into surgically created tendon defects is safe and effective. MSCs improved the tendon's maximum load over time, indicating that MSCs could help facilitate the dynamic process of tendon repair.
\end{abstract}

Keywords: Mesenchymal stem cells (MSCs), Animal model, Rotator cuff

\footnotetext{
* Correspondence: benjamin.fernandez@salud.madrid.org

${ }^{1}$ UGC de Reumatología, Hospital Clínico San Carlos, Instituto de Investigación

Sanitaria del Hospital Clínico San Carlos (IdISSC), Madrid, Spain

Full list of author information is available at the end of the article
} 


\section{Background}

The rotator cuff is formed by a group of muscles and tendons that stabilise the glenohumeral joint and enable the normal movement of the shoulder. Rotator cuff tears can occur due to traumatic injury, but most occur as a result of the gradual degeneration of the tendon. Consequently, elderly individuals are often affected in a chronic manner. Partial and/or full thickness tears in the rotator cuff tendons are common causes of pain and disability of the shoulder and can ultimately lead to osteoarthritis.

The surgical procedures that are typically used to treat these lesions, which use sutures to reattach the torn tendons to the bone, are unreliable, and lesion reoccurrence is very frequent reaching more than $50 \%$ [1]. Due to the weakness of the fibres in the affected tendon, primarily the supraspinatus (SP) tendon, at its insertion site, it is very difficult to correctly reattach the tendon during surgery [2]. Clinical failure in degenerative lesions has been linked to preoperative factors such as age [3], reduced acromiohumeral distance [4], tear size [5-8], chronically inferior tissue quality and tendon retraction and to surgical factors such as high repair tension [9]. All of these factors have a marked effect on biological healing and remodelling, which eventually influence the clinical outcome. When full-thickness tears occur in the elderly, in whom the natural healing ability is greatly reduced, it is crucial to reduce the in vivo mechanical forces that work against tendon repair during postoperative healing and to enhance natural healing, thereby preventing tendon re-ruptures and eventually resulting in a better clinical outcome. Therefore, there is a need for new approaches that improve the healing process through mechanical re-enforcement and enhance natural healing, especially at the insertion site. In this context, different therapies in the field of tendon regeneration, such as the local injection of mesenchymal stem cells (MSCs) and growth factors or tissue engineering using bioactive biological membranes, have been tested in different animal models [10-16]. These approaches have been reported to improve tendon repair, although the complete regeneration of the tendon has never been achieved. Although the research in this field is intense, the majority of studies have focused on the treatment of traumatic injuries, especially in young animals, in which the muscle and tendon are not as degraded as the tissue in chronic lesions [17]. As a result of these differences, these animal studies report better outcomes than are likely to occur in humans. In the current study, we developed and validated a massive chronic rotator cuff tear rat model to explore different repair strategies. We compared three different strategies: a surgical procedure based on a modified Masson-Allen stitch using a suture, a surgical procedure using a commercially available biocomposite scaffold consisting of type I collagen that is available for human use and a surgical procedure using the type I collagen scaffold in combination with allogeneic MSCs.

\section{Materials and methods \\ Study design}

Sprague-Dawley rats (9 months old) were included in this study. The three therapeutic approaches were performed 1 month postdetachment of the SP tendon and are described as follows: (a) a surgical procedure based on a modified Masson-Allen stitch using a suture (6/0 prolene; $n=12$ ), (b) a surgical procedure using a $0.5-\mathrm{mm}$-thick commercial type I collagen membrane (OrthADAPT ${ }^{\circ}$ Bioimplant, Penta Biomedical ${ }^{\circ}$ S.r.l., Verona, Italy; $n=15$ ) and (c) a surgical procedure using the type I collagen membrane seeded with $1 \times 106$ MSCs $(n=14)$.

\section{Validation of experimental rat model of chronic rotator cuff tears}

To generate chronic rotator tears in the animal model, a tear was surgically created in one of the shoulders by sharply detaching the SP tendon from its insertion site. While adhering to aseptic operating room protocols, the deltoid muscle was subsequently detached sharply from the acromion. The SP was visualised and then exposed by external rotation of the humerus, and it was detached sharply at the bone insertion site. The end of the tendon was marked with a suture (5/0 prolene) with a long tail. The fibrocartilage was removed from the insertion site with a scalpel blade, and the SP tendon was allowed to freely retract. The overlying deltoid muscle and skin were then closed (Fig. 1). Rats were sacrificed at months $1(n=16), 2$ $(n=13)$, and $3(n=12)$ postinjury, and specimens (humerus and scapula) were harvested. Recovery was evaluated through biomechanical evaluations. Animal care was performed in accordance with institution guidelines. The approval was given by the Ethics Committee of the Hospital Clinico San Carlos.

\section{Different approaches for the repair chronic rotator cuff tears in the rat model}

One month after the injury, one of three different additional surgeries was performed to repair the tears: (a) the typical surgical procedure using a suture (6/0 prolene), (b) a surgical procedure using a 0.5 -mm-thick commercial type I collagen membrane (OrthADAPT ${ }^{\circ}$ Bioimplant, Penta Biomedical $^{\circ}$ S.r.l., Verona, Italy), and (c) a surgical procedure using a type I collagen membrane seeded with $1 \times 106$ MSCs. Scar tissue from the first surgery was removed before the second surgical procedure was performed. The rats were sacrificed at 1, 2, and 3 months postinjury, and specimens (humerus and scapula) were harvested. Recovery was evaluated at the time of sacrifice by biomechanical analyses. 


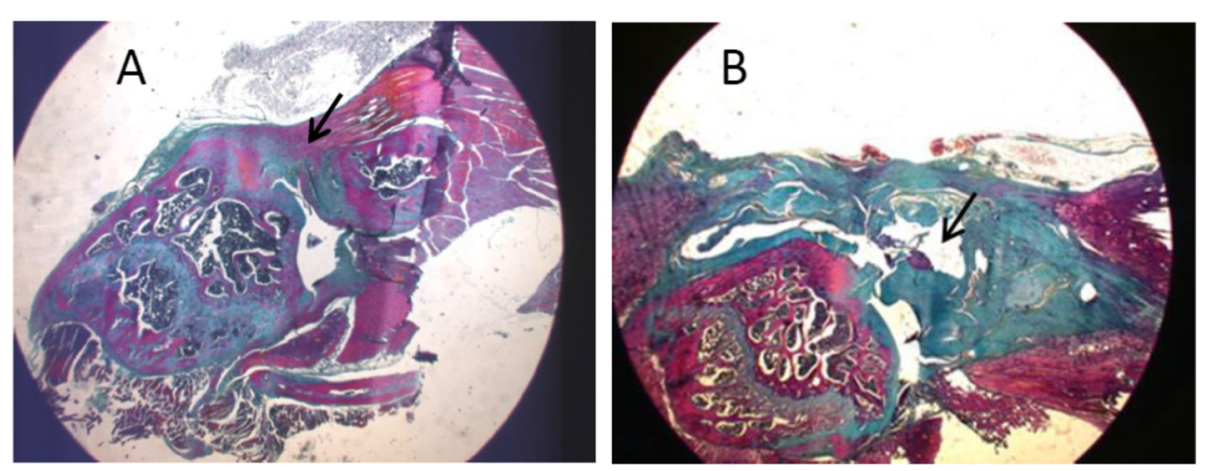

Fig. 1 Representative image of established chronic supraspinatus lesion. Black arrows represent the tendon-bone interface. a Gap is clearly observed in b magnification $\times 125$. a Normal tendon. $\mathbf{b}$ Injured tendon

\section{Cells and type I collagen membrane (OrthADAPT) preparation}

MSCs were obtained from the femoral channel of agematched rats (9 months old) prior to the study. The cells were placed in $100-\mathrm{mm}$ dishes, and the cultures were maintained for $48 \mathrm{~h}$ under standard conditions. MSCs adhered to the plates and proliferated to form colonies. When the cultures reached confluence, the MSC colonies that adhered to the plate were detached using $0.25 \%$ Trypsin-EDTA (Gibco BRL Life Technologies, Carlsbad, CA, USA). The cells were counted, and their viability was determined using the Trypan blue exclusion method. Aliquots containing $1 \times 106$ cells were suspended in $200 \mu \mathrm{l}$ of saline. OrthADAPT membranes were placed on the cell suspension containing $1 \times 10^{6}$ cells MSCs within 15 min before the event of surgical procedure. Previously, we have established that $5 \mathrm{~min}$ is the time the cells need in order to be adherent on the surface. No MSCs were observed in the remaining saline liquid.

\section{Biomechanical analysis}

Specimens (humerus and scapula) were dissected and stored at $-20{ }^{\circ} \mathrm{C}$ until analysis. For the mechanical testing, both sides of the tendon (humerus and scapula) were wrapped tightly with elastic rubber to prevent the sample from slipping out of the tube during the tensile test. For additional support, the rubber was fixed to the sample smear on both ends with the use of superglue. The tendons were wrapped in gauze and moistened with physiological serum to protect them. Two sets of tubes were prepared for the attachment of the samples to tensile machine grips: smaller tubes made of silicone (15 mm diameter) for the humerus and larger tubes made of polypropylene ( $25 \mathrm{~mm}$ diameter) for the scapula. One end of each tube was sealed with a piece of foil to prevent leakage of the resin. To prepare the resin, we mixed two types of glue (Araldite, Huntsman Advanced Materials, Basel, Switzerland) in equal proportions (50/
$50 \%)$. Afterwards, the resin was poured into the smaller tube in which the humerus was placed, being careful to support the other end of the supraspinatus complex (scapula) to preserve the physiological angle between the humerus and scapula. We then prepared the resin that was poured into the larger tube, which contained the scapula, in the same manner. The specimens were tested parallel to the long axis of the SP tendon in their physiological position using an Instron 5866 electromechanical testing instrument. The velocity used during testing was $1.8 \mathrm{~mm} / \mathrm{min}$. The samples were loaded up to $10 \mathrm{~N}$ during the test, unloaded to the initial point and then loaded again until they reached the failure point. To estimate the stiffness of the tendons, the tensile strength machine registered the load $[\mathrm{N}]$ and displacement $[\mathrm{mm}]$. The repaired stiffness was defined as the slope of the load divided by the displacement at the failure point. Failure was defined as the maximum value of the load needed to permanently damage the sample.

\section{Statistical analysis}

Continuous variables were described using mean and standard deviation (SD). To analyse the effect of the different surgical treatments in the repaired tendons' biomechanical capabilities (maximum load, stiffness and deformity), a two-way ANOVA model was used, introducing an interaction between such factor and time (1, 2 and 3 months postinjury). Simple effects analysis, adjusted by Sidak's method, was used to study both the effects of time at each treatment level and the effects of treatment at each time. Effects size was calculated using Cohen's $f$.

A priori sample size calculation was performed using G*Power (Heinrich-Heine-University Düsseldorf), assuming that the interaction between time (1 and 3 month) and the treatment (repair with OrthADAPT and MSCs) compared with the use of suture had a large effect size (Cohen's $f=0.40$ ), a two-tailed type I error of 0.05 , and an $80 \%$ power. Based on such values, we would need a total sample 
size of 52 animals. Also, a post hoc statistical power calculation was performed. Statistical analyses were performed using STATA v12.

\section{Results}

Macroscopic examinations of the specimens

Gross examinations of the specimens at the time of dissection at 3 months after the repair treatment revealed scar tissue around the healed area with inflammation signs in all the study groups. Nevertheless, the signs of inflammation were seen to be more prominent in animals treated with suture or collagen membrane in contrast with rats treated with MSCs. The treatment with MSCs provided a clearer view of the repair area indicative of a minor scar inflammatory condition (Fig. 2).

\section{Different strategies for the repair of injured SP tendons}

Biomechanical tests of the repaired SP tendons are summarised in Table 1. With regard to maximum load, we observed an almost significant interaction between treatment and time $(F=2.62, d f=4, p=0.053)$. Effect size for such interaction was large (Cohen's $f=0.41$ ), and therefore, we decided to perform simple effects analysis, despite this lack of significance, to better characterise the relationship between both factors and their effect in maximum load. When we analysed how this biomechanical capability changed with time for each treatment, we observed that repair with OrthADAPT and MSCs was associated with a significant increase in maximum load $(p=0.04)$, between months 1 and 3 (Table 2). On the other hand, when we compared the different treatments among themselves at different time points, we observed that the repair with OrthADAPT and MSCs has associated with a significant higher maximum load, when compared with the use of suture, but only at 3 months $(p=0.014)$ (Table 3). With regard to stiffness and deformity, no significant interaction was observed $(F=1.68$, $d f=4, p=0.18 ; F=0.40, d f=4, p=0.81$; respectively).
In the post hoc statistical power calculation, based on the observed results, our study achieved a $70 \%$ power.

\section{Discussion}

Despite the great advances in the surgical procedures used to treat rotator cuff tears, the frequency of rerupture is still unacceptable for large injuries. Although the cause of this poor healing rate is not well known, chronic degenerative lesions are most common in the elderly, and the elevated tension to which the suture is subjected makes determining the prognosis for healing decidedly more complicated [5-7]. Therefore, the first aim of this study was to develop an animal model to reproduce, as much as possible, the conditions observed in elderly patients who have rotator cuff tears. This model had to take into account key aspects such as age, the chronic nature of the injury and full-thickness injury to the SP tendon. Once the model was established, our second aim was to reproduce SP tendon detachment and delayed repair to mimic the repair of chronic rotator cuff tears in humans. We used three different repair strategies-sutures and type I collagen membranes with or without MSCs-to determine the most suitable treatment for the repair of chronic rotator cuff tears. Soslowsky's group [9] have established rat models of chronic rotator cuff tears; however, the biomechanical properties of these models were not the same. Our rat model, in which we detached the SP tendon, is similar to that proposed by Soslowsky, but in contrast to the results for Soslowsky's model, in the present study, the failure to load and stiffness progressively decreased with time postdetachment. This trend is most likely due to the erratic formation of scar tissue adhesions and their subsequent degradation over time.

Whereas most animal models have been developed in young animals, the current study used older animals to develop a chronic rotator cuff tear rat model in which
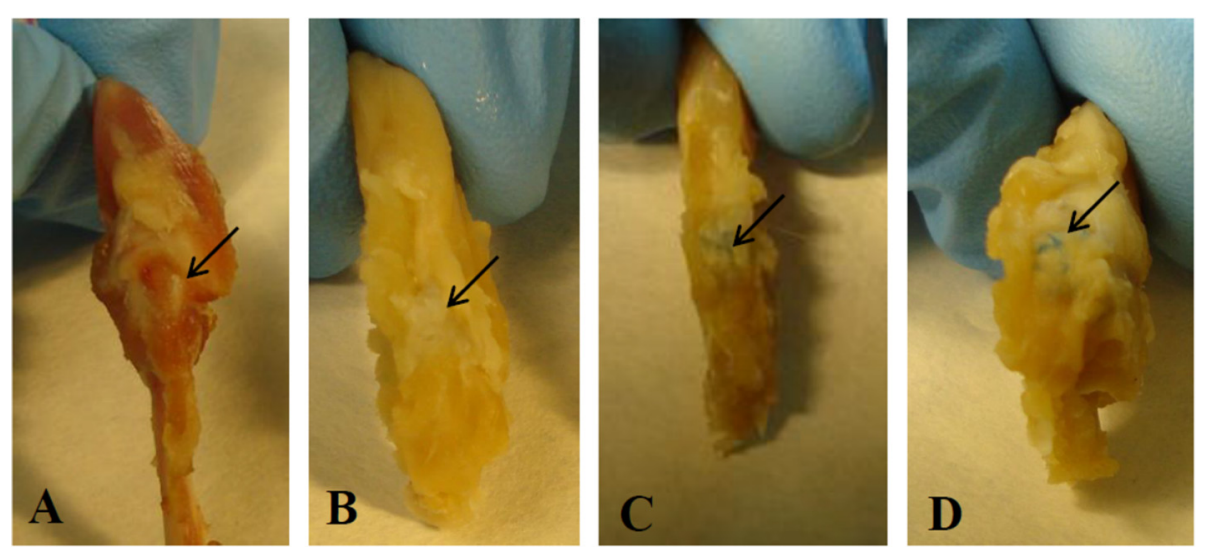

Fig. 2 Macroscopic view of shoulders. a Normal shoulder. b Suture. c Suture plus collagen membrane. d Suture plus collagen membrane plus mesenchymal stem cells. Black arrows represent the tendon-bone interface 
Table 1 Biomechanical characteristics

\begin{tabular}{|c|c|c|c|c|c|c|c|}
\hline & & $n$ & Suture mean (SD) & $n$ & OrthADAPT mean (SD) & $n$ & OrthADAPT + MSCs mean (SD) \\
\hline \multicolumn{8}{|l|}{ Max. load } \\
\hline & 1 & 4 & $34.84(6.96)$ & 6 & $24.16(15.81)$ & 6 & $25.27(9.96)$ \\
\hline \multirow[t]{2}{*}{ Time (months) } & 2 & 4 & $29.77(10.81)$ & 4 & $32,59(15.65)$ & 5 & $27.86(11.1)$ \\
\hline & 3 & 4 & $20.63(11.69)$ & 5 & $31.43(6.97)$ & 3 & $45.01(1.84)$ \\
\hline \multicolumn{8}{|l|}{ Deformation } \\
\hline & 1 & 4 & $7.5(2.3)$ & 6 & $6.13(2.72)$ & 6 & $10.08(9.37)$ \\
\hline \multirow[t]{2}{*}{ Time (months) } & 2 & 4 & $10.08(2.24)$ & 4 & $8.92(2.97)$ & 5 & $7.92(5.21)$ \\
\hline & 3 & 3 & $8.01(3.42)$ & 5 & $7.82(7.17)$ & 4 & $7.9(3.42)$ \\
\hline \multicolumn{8}{|l|}{ Stiffness } \\
\hline & 1 & 4 & $4.83(0.94)$ & 6 & $3.03(1.66)$ & 6 & $2.58(1.26)$ \\
\hline \multirow[t]{2}{*}{ Time (months) } & 2 & 4 & $3.03(1.02)$ & 4 & $5.33(4.13)$ & 5 & $2.82(1.12)$ \\
\hline & 3 & 3 & $2.63(1.06)$ & 5 & $5.56(4.11)$ & 4 & $4.45(0.57)$ \\
\hline
\end{tabular}

Max. load maximum load, SD standard deviation, MSCs mesenchymal stem cells

the healing capacity is reduced, which might account for the lack of spontaneous healing. These results were confirmed by the presence of degenerative changes in the surrounding tissue, such as adipose tissue infiltrating the muscle, and mineral changes affecting the humeral head, which were observed macroscopically during dissection. Overall, our animal model showed decreased mechanical functioning as well as degenerative histological changes consistent with a chronic condition. The loss of strength as time progressed after treatment mimics the long-term high re-rupture rate observed in humans after treatment with primary surgery.

The ability of the MSCs to improve the biomechanical characteristics of the injured SP tendon compared with the other two strategies was encouraging. While the suture-treated animals showed a loss of biomechanical functioning as time progressed, in the MSC-membrane repair group, the maximum load to failure was statistically significantly improved at 3 months compared with suture groups. This finding indicates that the animals treated with MSCs have greater strength in the injured SP in terms of the resistance to breakage, which is consistent with the results of previous studies reporting significant increases in biomechanical functioning after implanting MSC collagen membranes in patellar tendon defects [18].

Table 2 Biomechanical characteristics

\begin{tabular}{llll}
\hline & Suture & OrthADAPT & OrthADAPT + MSCs \\
\hline 2 vs. 1 month & 0.89 & 0.57 & 0.97 \\
3 vs. 1 month & 0.21 & 0.64 & $0.04^{*}$ \\
3 vs. 2 months & 0.58 & 0.99 & 0.11 \\
\hline
\end{tabular}

Maximum load by time

MSCs mesenchymal stem cells

${ }^{*} p<0.05$
In summary, our study demonstrated that tendon treatment with MSCs significantly improved the tendon's maximum load property and resulted in an augmented remodelled appearance compared with the other two methods at 3 months. It is possible that the healing process in the healthy state of the tendon is slowed, which could be explained by the animal model used in this study. First, to mimic the repair of chronic rotator cuff tears in humans, we began treatment in our animal model 1 month after detachment. It is known that healing is worse under these conditions than in acute injuries, although the reasons remain unclear. Second, MSCs were obtained from aged animals, which possess a decreased natural healing capacity. Although the exact mechanism responsible for the positive influence of MSCs on tendon healing remains uncertain, it is well known that MSCs have the capacity to differentiate into tenocytes, possess the capability to release growth factors and exhibit anti-inflammatory effects [19] at the site of injury, which could help provide a better healing environment and eventually lead to the regeneration of the damaged tissue.

Given the age of the rats used in the current study, the MSC differentiation rate might be slower, or these cells could even release growth factors in a slower manner than MSCs isolated from younger animals. We therefore

Table 3 Biomechanical characteristics

\begin{tabular}{llll}
\hline & 1 month & 2 months & 3 months \\
\hline OrthADAPT vs. suture & 0.37 & 0.98 & 0.39 \\
OrthADAPT + MSCs vs. suture & 0.47 & 0.99 & $0.014^{*}$ \\
OrthADAPT + MSCs vs. OrthADAPT & 0.99 & 0.90 & 0.27 \\
\hline
\end{tabular}

Maximum load by different approaches for the repair

MSCs mesenchymal stem cells

${ }^{*} p<0.05$ 
believe that it is important to explore the effect of MSCs over a longer treatment period and to assess MSCs isolated from donors of various ages, especially in the case of aged animals.

In a rotator cuff tear model developed in young animals, Gulotta et al. [20] reported that there was a lack of differences with regard to the strength of the tendon and the alignment of the tendon collagen fibres when the animals were injected with MSCs compared with the vehicle-injected controls at 1 month, but these researchers neglected to evaluate the animals at later time points. However, they reported an improvement in the biomechanical and morphological properties in young animals at 45 days after the injection of MSCs.

Our findings suggest that the therapeutic use of allogeneic MSCs in type I collagen membranes for the repair of rotator cuff tears appears to be the most promising treatment in the long term. Nevertheless, questions concerning the optimal MSC dose, the effective time period, the optimal type of matrix material and donor age remain unanswered and will need to be addressed in future studies. This knowledge will hopefully lead to further advances that will improve the regeneration of injured SP tendons in the rotator cuff.

\section{Conclusions}

Gross inspection of the specimens at the time of dissection revealed that the musculoskeletal unit was stiff, and the tendon appeared to be retracted. Although some studies using animal models have reported that the tendon heals naturally after release, producing a neotendon even in the absence of a surgical procedure using sutures, the aged rats used as our animal model retained the gap even at 3 months after the infliction of the lesion. The introduction of MSCs in the type I collagen membrane placed at the site of the surgically created defects resulted in the production of mechanically superior tissue, with improved SP tendon strength and stiffness at 3 months after treatment in comparison with the use of an acellular collagen membrane or sutures. More work is needed to determine the optimal matrix and dose of MSCs. The improvement is most likely influenced by mechanisms that affect the differentiation of MSCs and by factors that control the inflammatory environment at the site of injury. The application of MSCs to treat rotator cuff injuries may help improve the functionality of the shoulder. Future studies will be needed to test the effect of MSCs on tendon healing over a prolonged period of time.

\section{Competing interests}

The authors declare that they have no competing interests.

\section{Authors' contributions}

PTE conceived of the rat model and general design and participated in the writing of the manuscript. JAH conceived of the rat model and participated in the surgery and histological analysis. EV was involved in the culture of MSCs. CRB, YLM, and FM participated in the surgery. YLG participated in the histological analysis. FJR, GVG, and AP participated in the biomechanical testing. LRR was involved in the statistical analysis. BFG was involved in the general design and writing of the manuscript. All authors read and approved the final manuscript.

\section{Acknowledgements}

The authors gratefully acknowledge financial support for this work provided by the Ministerio de Ciencia e Innovación (Spain) (Grant \# MAT 2009-10258), by Comunidad de Madrid (Grant \# S2011/BMD-2460) by Fundación Marcelino Botín. This work has been also financed by red RIER, FIS 10/00178 and EC10-061 from the Ministerio de Ciencia e Innovación (Spain). This work is part of a doctoral thesis 'Supraspinatus tendon regenerative therapy: study in a murine model of chronic injury' by José Antonio Fernández Hoyas.

\section{Author details}

${ }^{1}$ UGC de Reumatología, Hospital Clínico San Carlos, Instituto de Investigación Sanitaria del Hospital Clínico San Carlos (IdISSC), Madrid, Spain. ${ }^{2}$ Dpto de Medicina y Cirugía Experimentales, Hospital Clínico San Carlos, Instituto de Investigación Sanitaria del Hospital Clínico San Carlos (IdISSC), Madrid, Spain. ${ }^{3}$ Dpto de Anatomía y Embriología Humana I, Facultad de Medicina Universidad, Complutense de Madrid, Madrid, Spain. ${ }^{4}$ Dpto de Ciencia de Materiales, Universidad Politécnica de Madrid, Madrid, Spain. ${ }^{5}$ Centro de Tecnología Biomédica, Universidad Politécnica de Madrid, Pozuelo de Alarcón, Madrid, Spain. 'Servicio de Cirugía Ortopédica y Traumatología, Hospital Clínico San Carlos, Instituto de Investigación Sanitaria del Hospital Clínico San Carlos (IdISSC), Madrid, Spain.

Received: 27 January 2015 Accepted: 5 August 2015

Published online: 14 August 2015

\section{References}

1. Bishop J, Klepps S, Lo IK, Bird J, Gladstone JN, Flatow EL. Cuff integrity after arthroscopic versus open rotator cuff repair: a prospective study. J Shoulder Elbow Surg. 2006;15:290-9.

2. Galatz LM, Griggs S, Cameron BD, lannotti JP. Prospective longitudinal analysis of postoperative shoulder function: a ten-year follow-up study of full-thickness rotator cuff tears. J Bone Joint Surg Am. 2001;83:1052-6.

3. Bjornsson HC, Norlin R, Johansson K, Adolfsson LE. The influence of age, delay of repair, and tendon involvement in acute rotator cuff tears: structural and clinical outcomes after repair of 42 shoulders. Acta Orthop. 2011;82:187-92.

4. Saupe N, Pfirrmann CW, Schmid MR, Jost B, Werner CM, Zanetti M. Association between rotator cuff abnormalities and reduced acromiohumeral distance. AJR Am J Roentgenol. 2006;187:376-82.

5. Goutallier D, Postel JM, Gleyze P, Leguilloux P, Van Driessche S. Influence of cuff muscle fatty degeneration on anatomic and functional outcomes after simple suture of full-thickness tears. J Shoulder Elbow Surg. 2003;12:550-4.

6. Cofield RH, Parvizi J, Hoffmeyer PJ, Lanzer WL, Ilstrup DM, Rowland CM. Surgical repair of chronic rotator cuff tears. A prospective long-term study. J Bone Joint Surg Am. 2001;83-A:71-7.

7. Bartolozzi A, Andreychik D, Ahmad S. Determinants of outcome in the treatment of rotator cuff disease. Clin Orthop Relat Res. 1994;308:90-7.

8. Hersche $\mathrm{O}$, Gerber C. Passive tension in the supraspinatus musculotendinous unit after long-standing rupture of its tendon: a preliminary report. J Shoulder Elbow Surg. 1998;7:393-6.

9. Gimbel JA, Van Kleunen JP, Lake SP, Williams GR, Soslowsky LJ. The role of repair tension on tendon to bone healing in an animal model of chronic rotator cuff tears. J Biomech. 2007;40:561-8.

10. Nourissat G, Diop A, Maurel N, Salvat C, Dumont S, Pigenet A, et al. Mesenchymal stem cell therapy regenerates the native bone-tendon junction after surgical repair in a degenerative rat model. PLoS One. 2010;5, e12248.

11. Kim SH, Chung SW, Oh JH. Expression of insulin-like growth factor type 1 receptor and myosin heavy chain in rabbit's rotator cuff muscle after injection of adipose-derived stem cell. Knee Surg Sports Traumatol Arthrosc 2013 Jun 5. [Epub ahead of print] 
12. Oliva F, Osti L, Padulo J, Maffulli N. Epidemiology of the rotator cuff tears: a new incidence related to thyroid disease. Muscles Ligaments Tendons J. 2014;4(3):309-14. eCollection 2014.

13. Manning CN, Kim HM, Sakiyama-Elbert S, Galatz LM, Havlioglu N, Thomopoulos S. Sustained delivery of transforming growth factor beta three enhances tendon-to-bone healing in a rat model. J Orthop Res. 2011;29:1099-105.

14. Gulotta LV, Kovacevic D, Packer JD, Deng XH, Rodeo SA. Bone marrowderived mesenchymal stem cells transduced with scleraxis improve rotator cuff healing in a rat model. Am J Sports Med. 2011;39:1282-9.

15. Longo UG, Lamberti A, Petrillo S, Maffulli N, Denaro V. Scaffolds in tendon tissue engineering. Stem Cells Int. 2012;2012:517165.

16. Santoni BG, McGilvray KC, Lyons AS, Bansal M, Turner AS, Macgillivray JD, et al. Biomechanical analysis of an ovine rotator cuff repair via porous patch augmentation in a chronic rupture model. Am J Sports Med. 2010;38:679-86.

17. Plate JF, Brown PJ, Walters J, Clark JA, Smith TL, Freehill MT, et al. Advanced age diminishes tendon-to-bone healing in a rat model of rotator cuff repair. Am J Sports Med. 2014;42:859-68.

18. Awad HA, Boivin GP, Dressler MR, Smith FN, Young RG, Butler DL. Repair of patellar tendon injuries using a cell-collagen composite. J Orthop Res. 2003:21:420-31.

19. Barry FP, Murphy JM, English K, Mahon BP. Immunogenicity of adult mesenchymal stem cells: lessons from the fetal allograft. Stem Cells Dev. 2005;14:252-65.

20. Gulotta LV, Kovacevic D, Ehteshami JR, Dagher E, Packer JD, Rodeo SA. Application of bone marrow-derived mesenchymal stem cells in a rotator cuff repair model. Am J Sports Med. 2009;37:2126-33.

\section{Submit your next manuscript to BioMed Central and take full advantage of:}

- Convenient online submission

- Thorough peer review

- No space constraints or color figure charges

- Immediate publication on acceptance

- Inclusion in PubMed, CAS, Scopus and Google Scholar

- Research which is freely available for redistribution 